(166)

\title{
Phytochemical Screening and Antimicrobial Activity of Extracts from Passiflora suberosa L. Leaves
}

\author{
Bandara K.R.V. ${ }^{1}$, Padumadasa C. $^{2}$ and Peiris L.D.C. ${ }^{*}$ \\ ${ }^{I}$ Department of Zoology, University of Sri Jayewardenepura, Sri Lanka \\ ${ }^{2}$ Department of Cemistry, University of Sri Jayewardenepura, Sri Lanka \\ *dinithi@sci.sjp.ac.lk
}

\begin{abstract}
Plants are the basis of traditional medicine system and have been the source of many of novel drug components. Passiflora suberosa is used in Sri Lankan Ayurvedic system to treat many diseases including diabetes. The objective of the present study was to evaluate phytochemical constituent of different extracts and antimicrobial effect of methanol and aqueous extracts of leaves of $P$. suberosa. Aqueous, methanol, chloroform and hexane extracts of leaves of $P$. suberosa obtained under reflux conditions were subjected for phytochemical screening according to previously established methods. Aqueous and methanol extracts of $P$. suberosa leaves possessed more phytochemicals, thus those extracts were subjected for antimicrobial study which was obtained using minimum inhibition assay as determined by agar well diffusion method. Both methanol and aqueous extracts ranging from $6 \mu \mathrm{g} / \mathrm{ml}-800 \mu \mathrm{g} / \mathrm{ml}$ were tested against both gram positive (Bacillus subtilis, Staphylococcus aureus and Enterococcus faecium) and gram negative bacteria (Pseudumonas aeruginosa, Salmonella typhimuriam and Escherichia cloi) while Gentamicin was used as the standarded drug. The phytochemical screening revealed the presence of saponins and anthraquinones in the chloroform extract, alkaloids, saponins, and flavonoids in the hexane extract and alkaloids, unsaturated sterols, triterpenes, saponins, flavonoids and tannins in both methanol and aqueous extracts. Proanthocyanidin, which is a potent free radical scavenger, was observed only in the aqueous extract. Further, only methanol extract was found to possess moderate activity against all the tested bacterial strains. Highest concentration $(800 \mu \mathrm{g} / \mathrm{ml})$ of methanol extract showed widest zone of inhibition $(7 \mathrm{~mm})$, indicating moderate activity against tested bacterial strains. In contrast, the aqueous extract showed poor activity against tested bacterial strains. In conclusion, results revealed the presence of bioactive natural compounds in aqueous and mehtanolic extracts that may be used in the development of pharmaceutical products. Similarly, preliminary studies on antimicrobial activity exhibited antimicrobial potential of methanol extract, which could be used as future antimicrobial sources for natural therapies, food industry.
\end{abstract}

Keywords: Passiflora suberosa, Phytochemical, Antimicrobial activity, Agar well diffusion

Proceedings of the International Forestry and Environment Symposium 2016, Department of Forestry and Environmental Science, University of Sri Jayewardenepura, Sri Lanka. 